\title{
Are there Strange Stars in the Universe?
}

\author{
Ignazio Bombaci \\ Dipartimento di Fisica, Università di Pisa, and INFN Sez. di Pisa, via \\ Buonarroti, 2, I-56127 Pisa, Italy
}

\begin{abstract}
Strange stars could represent a new class of stellar compact objects, where a new phase of dense matter (deconfined strange quark matter) comes into play. Here, we discuss some possible observational evidence for their existence.
\end{abstract}

\section{Introduction}

Strange stars (SS) are hypothetical astrophysical compact objects which are made entirely of deconfined $u, d, s$ quark matter (strange quark matter). The possible existence of SS is a direct consequence of the conjecture (Bodmer 1971, Witten 1984) that strange quark matter may be the absolute ground state of strong interacting matter rather than ${ }^{56} \mathrm{Fe}$. Since this hypothesis was formulated, strange stars have been studied by many authors, but they remained purely theoretical entities.

\section{Strange star candidates}

Recent studies have shown that the compact objects associated with the transient X-ray burster SAX J1808.4-3658 (Li et al. 1999a), the atoll X-ray source $4 \mathrm{U} 1728-34$ (Li et al. 1999b), the X-ray burster 4U 1820-30 (Bombaci 1997), the X-ray pulsar Her X-1 (Dey et al. 1998), and the hard X-ray burster GRO J174428 (Cheng et al. 1998) are good strange star candidates. If the compact objects in these X-ray sources are really strange stars, then there will be very deep consequences for both astrophysics and the physics of strong interactions.

As a particular example here we consider the case of the transient X-ray burst source SAX J1808.4-3658. This source contains a millisecond X-ray pulsar with a period of 2.49 milliseconds.

Using the observed X-ray luminosities, Li et al. (1999a) derived a firm upper limit on the radius $R$ of the compact object in SAX J1808.4-3658 given by the dashed curve in the mass-radius (MR) plane in Fig. 1. The dotted curve in Fig. 1 represents the Schwarzschild radius $2 G M / c^{2}$, which is a lower limit of the stellar radius to prevent the star collapsing to a black hole. Thus the allowed range of the mass and radius of SAX J1808.4-3658 is the region confined by the dashed and dotted curves in Fig. 1. In the same figure, we report the theoretical MR relations (solid curves) for neutron stars given by five recent realistic models for the EOS of dense matter. Models $\mathrm{UU}, \mathrm{BBB} 1$ and $\mathrm{BBB} 2$ are relative to 


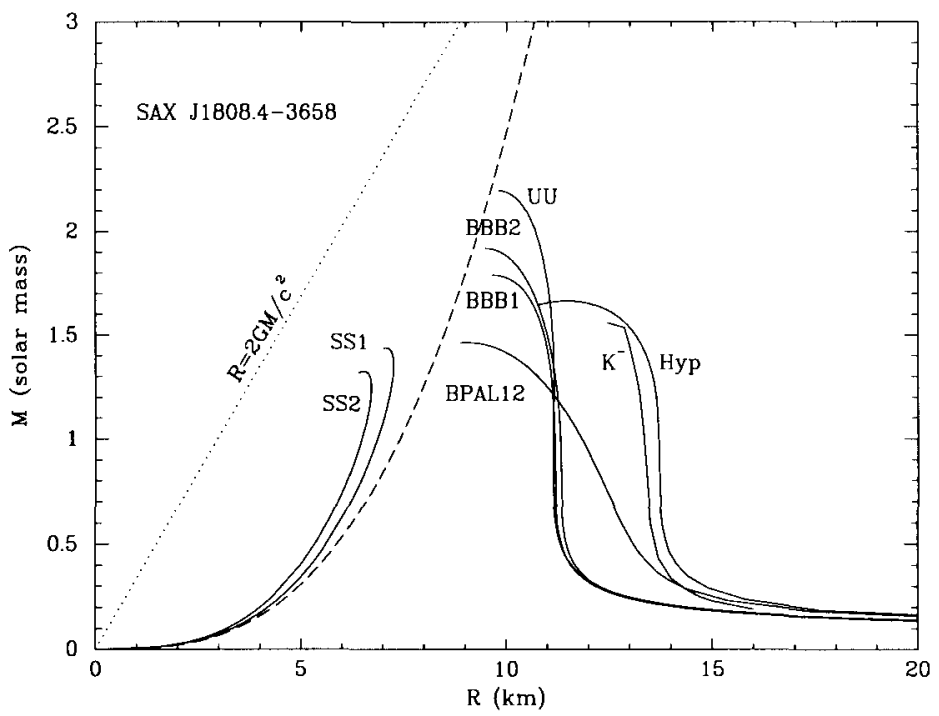

Figure 1. Comparison of the $M R$ relation of SAX J1808.4-3658 determined from Rossi X-ray Timing Explorer observations with theoretical models of neutron stars and of strange stars. See text for more details.

"conventional" neutron stars. The curve labeled Hyp depicts the MR relation for a neutron star in which hyperons are considered in addition to nucleons as hadronic constituents. It is clearly seen in Fig. 1 that none of the neutron star MR curves is consistent with SAX J1808.4-3658. The two curves SS1 and SS2 in Fig. 1 give the MR relation for strange stars calculated with the EOS by Dey et al. (1998). Figure 1 clearly demonstrates that a strange star model is more compatible with SAX J1808.4-3658 than a neutron star one.

\section{References}

Bodmer, A. R. 1971, Phys. Rev. D, 4, 1601

Bombaci, I. 1997, Phys. Rev. C, 55, 1587

Cheng, K.S., Dai, Z.G., Wei, D.M. \& Lu, T. 1998, Science, 280, 407

Dey, M., Bombaci, I., Dey, J., Ray, S., \& Samanta, B. C. 1998, Phys. Lett. B, 438,123

Li, X.-D., Bombaci. I., Dey, M., Dey, J., \& van den Heuvel, E. P. J., 1999a, Phys. Rev. Lett., (in press), hep-ph/9905235

Li, X.-D., Ray S., Dey, J., Dey, M., \& Bombaci. I. 1999b, ApJ, (in press), astro-ph/9908274

Witten, E. 1984, Phys. Rev. D, 30, 272 